\title{
294.
}

\section{ON A NEW ANALYTICAL REPRESENTATION OF CURVES IN SPACE.}

[From the Quarterly Journal of Pure and Applied Mathematics, vol. v. (1862), pp. $81-86$.

THE employment of a new kind of coordinates for the analytical representation of curves in space is suggested in my former paper under the same title Journal, t. III., pp. 225-236 (1859). The idea was as follows: viz. if $(x, y, z, w)$ are current coordinates of a point in space (ordinary point coordinates), and $(\alpha, \beta, \gamma, \delta)$ the coordinates of a particular point, then taking $(p, q, r, s, t, u)$ to represent the minor determinants formed out of the matrix

viz.

$$
\left(\begin{array}{llll}
x, & y, & r, & w \\
\alpha, & \beta, & \gamma, & \delta
\end{array} \mid\right.
$$

$$
\begin{array}{ll}
p=\gamma y-\beta z, & s=\delta x-\alpha w, \\
q=\alpha z-\gamma x, & t=\delta y-\beta w, \\
r=\beta x-\alpha y, & u=\delta z-\gamma w
\end{array}
$$

values which satisfy identically

$$
p s+q t+r u=0,
$$

then the equation of a cone passing through a given curve and having for its vertex the arbitrary point $(\alpha, \beta, \gamma, \delta)$, is of the form

$$
V=0 \text {, }
$$

$V$ being a homogeneous function of the six new coordinates $(p, q, r, s, t, u)$. And it was proposed to consider $V=0$ as the equation of the curve.

But as remarked in the paper, it is not every function $V$ of the coordinates $(p, q, r, s, t, u)$ which equated to zero, does in fact represent a curve. In order that 
the equation $V=0$ may represent a curve, it is necessary, that when any infinitesimal variations whatever are given to the constants $(\alpha, \beta, \gamma, \delta)$, thus converting the equation into $V+\delta V=0$, the two equations $V=0, \delta V=0$ (considered as equations in ordinary point coordinates) shall represent one and the same curve, whatever the system of infinitesimal variations attributed to $\alpha, \beta, \gamma, \delta$ may be. Let $P, Q, R, S, T, U$ denote the differential coefficients of $V$ in regard to $p, q, r, s, t, u$ respectively, then the equation $\delta V=0$, breaks up into the equations

$$
\begin{array}{r}
-R y+Q z-S w=0 \\
R x \cdot P z-T w=0 \\
-Q x+P y-U w=0 \\
S x+T y+U z \quad=0
\end{array}
$$

and the system composed of these four equations and the equation $V=0$ (considered as equations in ordinary point coordinates) must belong to one and the same curve.

The four equations gave

$$
P S+Q T+R U=0
$$

a relation between the differential coefficients of $V$ which must be satisfied either identically or in virtue of the equation $V=0$. And this relation existing, any two of the four equations lead to the other two. Attending exclusively to the coordinates $(p, q, r, s, t, u)$ and considering $(x, y, z, w)$ as mere arbitrary multipliers, the above equation

$$
P S+Q T+R U=0
$$

is the only relation between the differential coefficients of $V$ which is deducible from the four equations.

But it was noticed that the equation $V=0$, even when $V$ is a function such that we have (identically or in virtue of the equation $V=0$ ) the equation $P S+Q T+R U=0$, does not of necessity represent a curve. Some further relation or relations between the differential coefficients of $V$ must therefore exist, either identically or in virtue of the equation $V=0$; and such relations can be found by resorting to the second differential $\delta^{2} V$ of the function $V$. In fact not only the equation $\delta V=0$ but the entire series of relations $\delta^{2} V=0, \delta^{3} V=0, \ldots$ should be satisfied by the coordinates of any point of the curve. I find by means of the equation $\delta^{2} V=0$ a plexus of equations, which are consequently necessary, and I am inclined to believe sufficient, in order that the equation $V=0$ may in fact represent a curve; the equations of the plexus are, it will be seen, very numerous, and certainly only a small number of them are independent, but this is a question which I have not as yet investigated.

Attending to the expressions for $p, q, r, s, t, u$, we have

$$
\begin{aligned}
& d_{\alpha}=\quad-y d_{r}+z d_{q}-w d_{s}=(1) \\
& d_{\beta}=x d_{r}-z d_{p}-w d_{t}=(2), \\
& d_{\gamma}=-x d_{q}+y d_{p}-w d_{u}=(3), \\
& d_{\delta}=x d_{s}+y d_{t}+z d_{u} \quad=(4),
\end{aligned}
$$


and writing for convenience $a, b, c, d$ instead of $d_{a}, d_{\beta}, d_{\gamma}, d_{\delta}$, we have

$$
d=(1) a+(2) b+(3) c+(4) d .
$$

It was in effect by operating on $V$ with this symbol and equating to zero the coefficients of $a, b, c, d$, that the before-mentioned equations

were found.

$$
\begin{array}{r}
-R y+Q z-S w=0 \\
R x-P z-T w=0 \\
-Q x+P y-U w=0 \\
S x+T y+U z \quad \cdot=0
\end{array}
$$

If to these equations we join the equation

$$
A x+B y+C z+D w=0
$$

where $A, B, C, D$ are arbitrary multipliers, we can express $x, y, z, w$ in terms of $A, B, C, D$ in such manner as to satisfy the four equations, viz. we have

$$
\begin{aligned}
& x=\quad B U-C T+D P \\
& y=-A U \cdot+C S+D Q \\
& z=A T-B S \cdot+D R \\
& w=-A P-B Q-C R
\end{aligned}
$$

and if in the expressions for (1), (2), (3), (4) we substitute for $x, y, z, w$ these values, and form therewith the value of $d$, which value $I$ will for distinction call $\mathfrak{D}$, we have

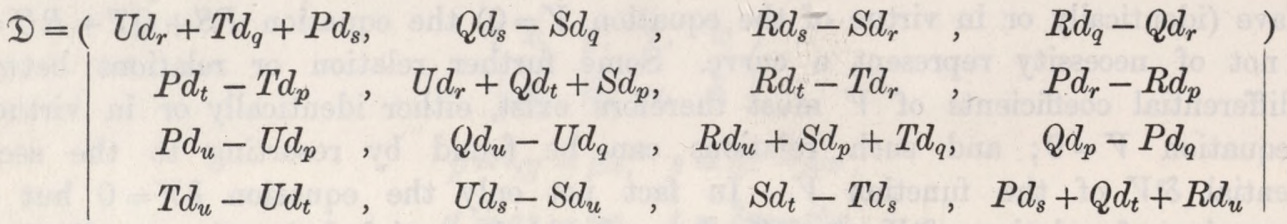

$$
\begin{aligned}
& (A, B, C, D)(\mathrm{a}, \mathrm{b}, \mathrm{c}, \mathrm{d}) \text {, }
\end{aligned}
$$

viz. $D$ is a lineo-linear function of the two sets of indeterminate quantities $(A, B, C, D),(\mathrm{a}, \mathrm{b}, \mathrm{c}, \mathrm{d})$, the coefficients thereof being the operators

$$
U d_{r}+T d_{q}+P d_{s}, Q d_{s}-S d_{q}, \& c .
$$

It may be remarked that we have identically

$$
\mathfrak{D V}=(P S+Q T+R U)(A \mathrm{a}+B \mathrm{~b}+C \mathrm{c}+D \mathrm{~d})
$$

since obviously each term such as $\left(Q d_{s}-S d_{q}\right) V$, which is equal to $Q S-S Q$, vanishes identically. The equation $\mathfrak{D} V=0$ gives therefore only the before-mentioned equation $P S+Q T+R U=0$, which is as it should be. 
The equation $\mathfrak{D}^{2} V=0$, is then to be satisfied independently of the values of $(A, B, C, D)$ and $(\mathrm{a}, \mathrm{b}, \mathrm{c}, \mathrm{d})$, and as $\mathfrak{D}$ contains 16 distinct terms, $\mathfrak{D}^{2}$ will contain in all $\frac{1}{2} 16.17$ or 136 distinct terms. The equation $\mathfrak{D}^{2} V=0$ gives therefore a plexus of 136 equations, and the equations in each succeeding plexus, involved in $\mathfrak{D}^{3} V=0$, $D^{4} V=0$, \&c. will, of course, be still be more numerous.

If $V=0$ be the plane conic which is the intersection of the surfaces

then we have

$$
\begin{aligned}
& x^{2}+y^{2}+z^{2}+w^{2}=0, \\
& a x+b y+c z+d w=0,
\end{aligned}
$$

$$
\begin{aligned}
& V=\left(b^{2}+c^{2},-a b,-a c, \quad . \quad c d,-b d\right)(p, q, r, s, t, u)^{2} . \\
& -b a, \quad c^{2}+a^{2}, \quad-b c,-c d, \quad . \quad, \quad a d \\
& -c a,-c b, a^{2}+b^{2}, \quad b d,-a d, \\
& \text {. , }-c d, \quad b d, a^{2}+d^{2}, \quad a b, \quad a c \\
& c d, \quad . \quad,-a d, \quad b a, b^{2}+d^{2}, \quad b c \\
& -b d, \quad a d, \quad . \quad c a, \quad c b, c^{2}+d^{2}
\end{aligned}
$$

The values of $P, Q, R, S, T, U$ (omitting a common factor 2) are

$$
\begin{aligned}
& P=\left(b^{2}+c^{2},-a b,-d^{i} c, .,+c d,-b d\right)(p, q, r, s, t, u), \\
& \text { \&c., }
\end{aligned}
$$

and if we proceed to form a term in $\mathscr{D}^{2} V$, say the coefficient of $A^{2} a^{2}$, this is $\left(U d_{r}+T d_{q}+P d_{s}\right)^{2} V$, or

$$
\left(a^{2}+b^{2}, c^{2}+a^{2}, a^{2}+d^{2},-c d,+b d,-c b\right)(U, T, P)^{2} .
$$

The coefficient therein of $p^{2}$ is

that is, it is

$$
\left(a^{2}+b^{2}, c^{2}+a^{2}, a^{2}+d^{2},-c d,+b d,-c b\right)\left(-b d, c d, b^{2}+c^{2}\right)^{2},
$$

$$
\begin{aligned}
& \left(a^{2}+b^{2}\right) b^{2} d^{2} \quad-2 c d . \quad c d\left(b^{2}+c^{2}\right) \\
+ & \left(c^{2}+a^{2}\right) c^{2} d^{2}+2 b d \cdot-b d\left(b^{2}+c^{2}\right) \\
+ & \left(a^{2}+d^{2}\right)\left(b^{2}+c^{2}\right)^{2}-2 c b \cdot-b d \cdot c d,
\end{aligned}
$$

where the terms in which $\left(b^{2}+c^{2}\right)$ does not appear as a factor are together equal to

$$
a^{2} d^{2}\left(b^{2}+c^{2}\right)+d^{2}\left(b^{2}+c^{2}\right)^{2},
$$

the entire expression thus divides by $b^{2}+c^{2}$, the quotient being

$$
\left(a^{2}+d^{2}\right)\left(b^{2}+c^{2}\right)-2 c^{2} d^{2}-2 b^{2} d^{2}+a^{2} d^{2}+d^{2}\left(b^{2}+c^{2}\right),
$$

which is equal to $a^{2}\left(b^{2}+c^{2}+d^{2}\right)$, or restoring the factor $b^{2}+c^{2}$, we see that in $\mathfrak{D}^{2} V$ the coefficient of $A^{2} a^{2}$ is

$$
a^{2}\left(b^{2}+c^{2}+d^{2}\right)\left(b^{2}+c^{2}\right) p^{2}+\& c .
$$


The complete value must, it is clear, be of the form

$$
a^{2}\left(b^{2}+c^{2}+d^{2}\right) V+k(p s+q t+r u)
$$

vanishing in virtue of the equations $V=0, p s+q t+r u=0$, and this being so, observing that $V$ contains no term in $p s$, we have $k=$ coefficient $p s$ in

$$
\left(a^{2}+b^{2}, c^{2}+a^{2}, a^{2}+d^{2},-c d,+b d,-c b\right)(U, T, P)^{2},
$$

that is

$$
k=2\left(a^{2}+b^{2}, c^{2}+a^{2}, a^{2}+d^{2},-c d,+b d,-c b\right)\left(-b d, c d, b^{2}+c^{2}\right)(c a, b a, 0),
$$

or

which is

$$
\begin{aligned}
\frac{1}{2} k= & \left(a^{2}+b^{2}\right) \cdot-b d \cdot c a-c d\left\{c d \cdot 0+\left(b^{2}+c^{2}\right) b a\right\} \\
& +\left(c^{2}+a^{2}\right) \cdot \quad c d \cdot b a+b d\left\{\left(b^{2}+c^{2}\right) c a-b d \cdot 0\right\} \\
& +\left(a^{2}+d^{2}\right) . \quad 0-c b\{-b d \cdot b a+c d \cdot c a\},
\end{aligned}
$$

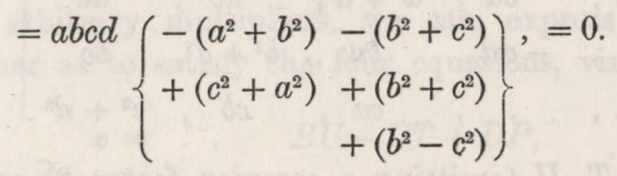

The coefficient $k$ consequently vanishes, and therefore in $\mathscr{D}^{2} V$ the coefficient of $A^{2} \mathrm{a}^{2}$ is $a^{2}\left(b^{2}+c^{2}+d^{2}\right) V$, but I have not worked out the coefficients of the other terms.

2, Stone Buildings, W.C., 30th October, 1860. 